\title{
Förderung der Integration durch Islamischen Religionsunterricht?
}

\author{
Klaus Spenlen
}

Religionsunterricht ist das einzige Schulfach mit Verfassungsrang, weshalb seine Berechtigung, Bestandteil des Fächerkanons auch in Zeiten zu sein, in denen immer wieder Forderungen laut werden, dass sich gesellschaftliche Entwicklungen in neuen Fächern konstituieren sollen (z. B. Gesundheits-, Verbraucher, Umweltunterricht), selbstverständlich hingenommen wird. Und das, obwohl sich in säkularen Gesellschaften wie der deutschen ein Abschmelzen konfessioneller Milieus abzeichnet, das in vielen Familien zu einem weitgehenden Verzicht auf religiöse Sozialisation führt. Damit verbunden ist eine Verringerung religiöser Prägekraft für Lebensstile mit der Folge, dass sich zunehmend mehr Kinder ihre Religiosität oder Religion außerhalb der Schule selbst „konstruieren" müssen. Verstärkt wird dieser Prozess durch einen zunehmenden Verlust von Identität zwischen Religionen, ihren Institutionen sowie Vertretern auf der einen und der Gesellschaft auf der anderen Seite, der religiöse Sinngebung und Weltdeutung verzichtbar erscheinen lässt.

Solche Entwicklungen scheinen in muslimischen Familien anders zu verlaufen, in denen religiöse Eltern ihr tradiertes Wissen und ihre religiösen Prinzipien an ihre Kinder weitergeben. Dabei sind die Erziehungsvorstellungen dieser Eltern durchweg geprägt von Erinnerungen an die eigene Kindheit sowie den Erwerb „eigenen religiösen Wissens durch die Islamdeutungen islamischer Gruppen, Medien und transnationaler Wissensnetzwerke ". ${ }^{1}$ Zwar sind muslimische Eltern i.d. R. keine religiösen Gelehrten im Sinne der ulamã, allerdings können nach muslimischem Verständnis auch Laien durch Praxiserfahrungen religiöse Expertise erwerben. In diese Expertise fließen dann Traditionen ein, die familiär und/oder regional geprägt sein können. Umso wichtiger erscheint

\footnotetext{
${ }^{1}$ Jeannette Spenlen, 2009, die sich auf eine Befragung muslimischer Eltern aus dem arabischen Kulturraum, die im Rheinland leben, beruft.
} 
es, muslimischen Kindern und Jugendlichen ihre Religion auf der Basis der islamischen Quellen von Koran und Hadîthe zu vermitteln, zumal Muslim zu sein für viele Jugendliche über den religiösen Bereich hinaus Lebensführung und Zukunftsorientierung bedeutet. ${ }^{2}$

Der Islam ist eine Religion der Orthopraxie, bei der es wesentlich auf Verhalten ankommt und der sich in Deutschland in der Diaspora befindet. Die ca. 4 Millionen Muslime in Deutschland, unter ihnen ca. 800.000 muslimische Schülerinnen und Schüler, haben die Diskussionen über das tradierte Verhältnis von Staat und Religion sowie Fragen nach gemeinschaftsbildenden Aufgaben von Religionen neu belebt und öffentlich gemacht. Dies führt zwangsläufig auch zu der Frage, wie lange es in den öffentlichen Schulen einer pluralistischen Gesellschaft noch konfessionellen Religionsunterricht geben kann, wie er sich - nicht nur verfassungsrechtlich - legitimiert und welches die Bedingungen für und die Grundlagen von schulischem islamischem Religionsunterricht sind.

\section{Verfassungsrecht}

Trotz der weit überdurchschnittlichen Aussagekraft der Texte der Religionsverfassung des Grundgesetzes (GG) weist kaum ein anderes Teilgebiet des Verfassungsrechts bei seiner Entwicklung in Praxis und Literatur solche Brüche und Dissonanzen auf wie das traditionelle Staatskirchenrecht. Deshalb wundert es nicht, dass grundrechtsdogmatisch heute u. a. Fragen der Zulässigkeit staatlicher Religionsförderung, des Kirchenvertragsrechts, des Körperschaftsstatus, des Grundrechtseingriffs und der Schranken der Religionsfreiheit mit ihren Einzelaspekten kontrovers und häufig mit gegenteiligen Einschätzungen diskutiert werden. ${ }^{3}$ In einem gestuften Verfahren steht die Anerkennung als Religionsgemeinschaft dabei an zweiter Stelle, nach dem Erwerb der Rechtsfähigkeit der Religionsgemeinschaft und vor der Anerkennung als Körperschaft des öffentlichen Rechts sowie weiteren Rechten, etwa der Vertretung in Rundfunkräten. ${ }^{4}$ Und was Juristen und Theologen in Deutschland unter einer Religionsgemein-

\footnotetext{
${ }^{2}$ Vgl. BMI 2007, S. 109 ff. sowie BMI 2009, S. 137 ff.

${ }^{3}$ Vgl. Gerhard Czermak, 2002, S. 2.

${ }^{4}$ Vgl. Ulrich Rhode, 2011, S. $111 \mathrm{ff}$.
} 
schaft verstehen, ist gesetzlich nicht festgelegt, wohl aber haben sich Gutachter und Gerichte dazu geäußert.

Der Verfassungsrang für schulischen Religionsunterricht ergibt sich aus Art. 7 Abs. 3, Sätze 1 und 2 GG:

Der Religionsunterricht ist in den öffentlichen Schulen mit Ausnahme der bekenntnisfreien Schulen ordentliches Lehrfach. Unbeschadet des staatlichen Aufsichtsrechtes wird der Religionsunterricht in Übereinstimmung mit den Grundsätzen der Religionsgemeinschaft erteilt.

Nach der häufig in der Rechtsliteratur verwendeten Umschreibung von Anschütz ${ }^{5}$ für die nahezu wortgleiche Vorgängernorm des Art. 149 Abs. 1 S. 3 der Weimarer Reichsverfassung (WRV) bedeutet dies, dass Religionsunterricht „in konfessioneller Positivität und Gebundenheit ${ }^{\text {“6 }}$ zu erteilen ist. Das Bundesverfassungsgericht (BVerfGE 74, 244, 252) hat Religionsunterricht als Verfassungsbegriff folgendermaßen definiert:

Er ist keine überkonfessionelle vergleichende Betrachtung religiöser Lehren, nicht bloß Morallehre, Sittenunterricht, historisierende und relativierende Religionskunde, Religions- oder Bibelgeschichte. Sein Gegenstand ist vielmehr der Bekenntnisinhalt, nämlich die Glaubenssätze der jeweiligen Religionsgemeinschaft. Diese als bestehende Wahrheiten zu vermitteln ist seine Aufgabe.

Damit hat das Gericht neben einer Positivdefinition zugleich Unterrichte beschrieben, die nicht Religionsunterricht im Sinne des GG sind, jedoch religiöse Themen zum Inhalt haben. Ausgenommen von der Pflicht für die Einführung von Religionsunterricht sind durch Artikel 141 GG Länder, in denen am 1. Januar 1949 eine andere landesrechtliche Regelung bestand. Auf diese sog. „Bremer Klausel" berufen sich Bremen, Berlin und Brandenburg. Mithin besteht in den dreizehn anderen Ländern bei Vorliegen der Voraussetzungen ein Rechtsanspruch auf Einführung von Religionsunterricht. ${ }^{7}$

Dabei handelt es sich um eine res mixta, d. h. um eine gemeinsame Angelegenheit von Landesregierung und Religionsgemeinschaft. Die Länder haben dafür

\footnotetext{
${ }^{5}$ Vgl. Gerhard Anschütz, 1933, Art. 137 Anm. 2.

${ }^{6}$ Die Verfassung des Deutschen Reiches vom 11. August 1919, S. 691.

${ }^{7}$ Vgl. Myrian Dietrich 2006, S. 48 ff., die die Komplexität des Rechtsanspruchs aufzeigt und u. a. der Frage nachgeht, ob die Religionsgemeinschaft, die Schülerinnen und Schüler oder deren Erziehungsberechtigte diesen Rechtsanspruch geltend machen können.
} 
die sachlichen und personellen Voraussetzungen zu schaffen und zu finanzieren, die letztlich der Erfüllung kirchlicher Aufgaben dienen (etwa Ausbildung der Lehrkräfte, Einrichtung von Lehrstühlen). Diese Aufgaben erfüllen die Länder, indem sie u. a. Personalausgaben, die bei einzelnen Religionsgemeinschaften anfallen, refinanzieren.

Auch wenn die Religionsgemeinschaften die Grundsätze des Religionsunterrichts festlegen und damit seinen Inhalt wesentlich bestimmen, bleibt er, wenn er als ordentliches Lehrfach an öffentlichen Schulen durchgeführt wird, staatlicher Unterricht, der nach Art. 7 Abs. 3 Satz 2 GG ausdrücklich der staatlichen Schulaufsicht unterliegt. Diese erschöpft sich nicht in der Aufsicht über die äuBeren Umstände des Unterrichts. Vielmehr kann der Staat für die Qualifikation der Lehrkräfte und die pädagogischen und wissenschaftlichen Standards Vorgaben machen und ihre Einhaltung sicherstellen. Darüber hinaus ist der staatlichen Schulaufsicht gem. Art. 7 Abs. 1 GG die Befugnis des Staates zu entnehmen, in den Grenzen der Verfassung eigene Erziehungsziele für das Schulwesen $\mathrm{zu}$ formulieren.

Ausdruck dieses Doppelstatus ist auch, dass in den Konkordaten mit der katholischen Kirche und den Staatskirchenverträgen mit den Evangelischen Kirchen eine kirchliche Einverständniserklärung bzw. ein konsultatives Votum der Kirchen vorgesehen ist. Vergleichbares gibt es derzeit im Hinblick auf den Islam nicht. In analoger Anwendung dieses Grundsatzes des Staatskirchenrechts wären von den Bundesländern mit islamischen Religionsgemeinschaften ggf. Staatsverträge zu schließen oder eine sonstige rechtsverbindliche Norm herbeizuführen. $^{8}$

\section{Islamische Religionsgemeinschaft}

Die gemeinsame zentrale politische Forderung der islamischen Verbände ist die Anerkennung des Islam als gleichberechtigte Religionsgemeinschaft in Deutschland, d. h. die Gleichstellung mit den christlichen Kirchen. Die Verbände möch-

\footnotetext{
${ }^{8}$ Den bevorstehenden Abschluss eines solches Staatsvertrages zwischen dem Senat der Stadt Hamburg und den drei islamischen Verbänden Schura (Rat der islamischen Gemeinschaften), DİTíB und dem VIKZ vermeldete erstmalig der NDR am 25.05.2012, unter: http://www.ndr.de/regional/hamburg/ staatsvertrag125.html. Der Abschluss steht zum Redaktionssschluss dieses Bandes Ende 2012 unmittelbar bevor.
} 
ten in Deutschland z. B. islamische Riten praktizieren, Moscheen und Friedhöfe bauen und islamischen Religionsunterricht (IRU) in Schulen erteilen dürfen.

Außer den vier islamischen Dachverbänden DİTİB (Türkisch-Islamische Union der Anstalt für Religion e. V. - Diyanet İşleri Türk İslam Birliği -), ZMD (Zentralrat der Muslime in Deutschland e. V.), Islamrat und VIKZ (Verein islamischer Kulturzentren in Deutschland e. V.) agiert noch der größte Dachverband der Aleviten in Deutschland, die „Almanya Alevi Birlikleri Federasyonu/ Alevitische Gemeinde Deutschlands e. V.“ (AABF). Aleviten unterscheiden sich in vielen Glaubensgrundsätzen, Riten und Gebräuchen von Muslimen. Es wird geschätzt, dass der Anteil von Aleviten an der türkischstämmigen Bevölkerung zwischen $20 \%$ und $30 \%$ ausmacht. Zudem versteht ein nicht näher zu quantifizierender Anteil von Aleviten ihre Religion als außerhalb des Islam. ${ }^{9}$

Um IRU einzuführen, beantragten der ZMD (bzw. dessen Rechtsvorgänger) 1994 sowie der Islamrat durch Beitritt 1996 beim damaligen Ministerium für Schule und Weiterbildung Nordrhein-Westfalen dessen Einführung. Nachdem die Anträge mit der Begründung, die Kläger stellten keine Religionsgemeinschaften dar, die Ansprechpartner des Landes für IRU seien, abgewiesen worden waren, folgte ein jahrelanger Rechtsstreit vor der Verwaltungsgerichtsbarkeit. ${ }^{10}$ In den Leitsätzen, mit denen die Klage abschließend abgewiesen wurde, führte das Bundesverwaltungsgericht $u$. a. aus, dass Dachverbände mehr sein müssen als das Sprachrohr der Mitgliedsvereine; dass sie die für die Identität der Religionsgemeinschaft wesentlichen Aufgaben auf Dachverbandsebene (religiöse Autorität) wahrnehmen müssen; dass eine Beschränkung auf die Vertretung gemeinsamer Interessen nach außen oder auf Koordinierung der Mitgliedsvereine nicht ausreichend sei und dass Mitgliedsvereine, die keine oder nur partiell religiöse Aufgaben wahrnehmen, den Dachverband nicht dominieren dürfen. Zudem scheide ein Verband als Partner des Staates bei der Einführung von Religionsunterricht aus, wenn er nicht die Gewähr biete, nicht gegen die fundamentalen Verfassungsprinzipien von Art. 79 Abs. 3 GG, die Grundrechte sowie die Grundprinzipien des Religions- und Staatskirchenrechts zu verstoßen.

\footnotetext{
${ }^{9}$ Vgl. die Beiträge von Ali Ertan Toprak und Ismail Kaplan (S. 273-303) in diesem Sammelband.

${ }^{10}$ Vgl. Urteile des VG Düsseldorf vom 02.11.2001 - VG 1 K 10519/98, des OVG Münster vom 02.12.2003 - OVG 19 A 997/02 sowie des BVerwG vom 23.02.2005 - BVerwG 6 C 2.04.
} 
Das Scheitern der Klage hat indes nichts mit dem Status der Kläger als Dachorganisation zu tun, denn auch eine bundesweite, einheitlich organisierte Religionsgemeinschaft kann gegenüber einem Land grundsätzlich die für die Einrichtung des Religionsunterrichtes in diesem Land erforderlichen Maßnahmen vornehmen. Die Gerichte verneinten bei den Dachverbänden „lediglich“ deren religiöse Kompetenz. Hinzu kommen beim Islamrat Vorbehalte wegen verfassungsrechtlicher Bedenken. ${ }^{11}$

An der Rechtssituation änderte auch die Gründung des Koordinierungsrates der Muslime (KRM) am 11. April 2007 durch DİTİ, ZMD, Islamrat und VIKZ juristisch nichts. Denn der bundesweit agierende KRM erfüllt nicht bereits durch den Zusammenschluss der vier Dachverbände die Voraussetzungen, die das BVerwG 2005 beim ZMD und Islamrat als nicht erfüllt angesehen hat. Zudem ist nach wie vor nicht erkennbar, ob es sich bei dem KRM lediglich um einen losen Zusammenschluss weiterhin selbstständiger Dachverbände oder um einen neuen Dachverband mit einer eigenen Rechtspersönlichkeit handelt.

Damit hatte nach wie vor keine der Landesregierungen, auf die Art. 7 Abs. 3 GG zutrifft, einen Partner für die flächendeckende Einführung von IRU an öffentlichen Schulen. Doch mit dem „Gesetz zur Einführung von islamischem Religionsunterricht als ordentliches Lehrfach (7. Schulrechtsänderungsgesetz) " vom 30.12.2011 hat der Landtag von NRW für die mehr als 320.000 muslimischen Schülerinnen und Schüler die Einführung von IRU vom Schuljahr 2012/13 an beschlossen. ${ }^{12}$ Wegen der besonderen Bedeutung des Religionsunterrichts für die Religionsfreiheit der Schülerinnen und Schüler sowie deren Eltern sollte seine Einführung nicht daran scheitern, dass die Qualifikation einer Organisation als Religionsgemeinschaft im Sinne von Artikel 14 und 19 Landesverfassung und Artikel 7 Absatz 3 GG noch nicht endgültig feststünde. ${ }^{13}$ In solchen Fällen sei es als Übergangslösung zu einem Religionsunterricht nach Art. 7 Abs. 3 GG denkbar, mit im Land verbreiteten Organisationen zu kooperieren, die Aufgaben wahrnähmen, welche für die religiöse Identität ihrer Mitglieder wesentlich sei-

\footnotetext{
11 Vgl. Urteil des OVG Rheinland-Pfalz vom 24. Mai 2005 - 7 A 10953/04.

${ }^{12}$ Vgl. Gesetz- und Verordnungsblatt (GV. NRW.), Ausgabe 2011 Nr. 34 vom 30.12.2011, Seite 725 bis 732 .

${ }^{13}$ Vgl. die Begründungen im Parteien übergreifenden Gesetzentwurf als LT-DS 15/2209 unter: http: //www.land-tag.nrw.de/portal/WWW/dokumentenarchiv/Dokument/MMD15-2209.pdf.
} 
en. Die Organisation müsse eigenständig und unabhängig sein und die Gewähr dafür bieten, dem Land bei der Veranstaltung des Religionsunterrichts auf absehbare Zeit als Ansprechpartner zur Verfügung zu stehen. Zudem müsse sie die in Artikel 79 Absatz 3 Grundgesetz umschriebenen Verfassungsprinzipien, die dem staatlichen Schutz anvertrauten Grundrechte der Schülerinnen und Schüler sowie die Grundprinzipien des freiheitlichen Religions- und Staatskirchenrechts des Grundgesetzes achten. Vertreten mehrere Organisationen das gleiche oder ein verwandtes Bekenntnis, soll das zuständige Schulministerium eine Zusammenarbeit mit ihnen gemeinsam anstreben. Damit verbinden die Parlamentarier die Erwartung, dass diese Organisationen innerhalb einer absehbaren Frist alle Merkmale einer Religionsgemeinschaft unzweifelhaft erfüllen.

Die fehlende Religionsgemeinschaft wird im Schulrechtsänderungsgesetz durch einen Beirat ersetzt, der wiederum innerhalb einer absehbaren Frist durch eine Religionsgemeinschaft ersetzt werden soll. Dieses Engagement der Politik schwankt zwischen der Ausgestaltung wohlwollender Neutralität und stärkerer Laizität. ${ }^{14}$ Aber auch mit der Variante Beirat steht bereits jetzt Ärger über Detailfragen ins Haus: Der Beirat besteht zur Hälfte aus Vertretern, die der KRM, und zur anderen Hälfte aus Vertretern, die das Ministerium benennt. Da auch DİTİB Mitglied des KRM ist, wird - neben dem deutschen Staat - einem ausländischen Staat, der Republik Türkei, proaktive Einflussnahme auf Bildungsinhalte in Deutschland eingeräumt.

Allerdings gibt es mit der Lösung, als vorläufigen Ansprechpartner der Landesregierung einen Beirat zu installieren, bereits Erfahrungen. So unterhält die Westfälische Wilhelms-Universität Münster seit mehreren Jahren am Centrum für Religiöse Studien einen Beirat, dem bei Bedarf Entscheidungsbefugnisse einer Religionsgemeinschaft zugebilligt werden. ${ }^{15}$ Vergleichbar arbeiten die Bei-

\footnotetext{
${ }^{14}$ Niedersachsen und Hessen beobachten den Versuch in NRW, weil auch sie beabsichtigen, möglichst bald IRU in ihren Ländern in ähnlicher Rechtskonstruktion einzurichten.

${ }^{15}$ Allerdings sind die Konditionen, die die Universität dem Beirat einräumt, schwer zu ertragen, weil sie den Mainstreamislam der konservativen Dachverbände damit perpetuiert und zugleich die Freiheit universitärer Lehre und Forschung massiv einschränkt: „Beanstandet der Beirat nachträglich die Lehrtätigkeit einer/eines angestellten oder berufenen Dozentin/Dozenten aus religiösen Gründen, so trägt die Westfälische Wilhelms-Universität dafür Sorge, dass die/der Betroffene nicht mehr im Bereich der Islamischen Theologie unterrichtet." Vgl. § 4 Abs. 2 der Ordnung des konfessionellen Beirats für Islamische Wilhelms-Universität Münster vom 21. Dezember 2011 unter:
} 
räte an denjenigen deutschen Universitäten, an denen Imame aus- und weitergebildet werden. Insofern bildet das Konstrukt Beirat bei der Implementation von IRU in NRW keine Ausnahme. Andererseits sind Schulen und Hochschulen insofern nicht miteinander zu vergleichen, als der universelle Anspruch des Staates an Religionsgemeinschaften auf Verfassungstreue in Bezug auf Schulen insbesondere das Verbot enthält, Schulkinder den Verfassungsprinzipien zu entfremden. Deshalb ist es ebenso konsequent wie unverzichtbar, dass der Landtag von NRW die Laufzeit des 7. Schulrechtsänderungsgesetzes bis Ende Juli 2019 befristet hat. Die Zeit bis dahin soll u. a. dafür genutzt werden, den Beirat durch eine Religionsgemeinschaft zu ersetzen, es sei denn, Verwaltungsgerichte werden vorher angerufen, um die Frage zu klären, ob ein Beirat überhaupt - wenn auch nur vorübergehend - Religionsgemeinschaft im Sinne von Art. 7 Abs. 3 GG sein kann.

\section{Erfahrungen aus dem staatlichen Islamkundeunterricht}

Verfassungsrechtliche Fragen zum Vorläufer von IRU, ${ }^{16}$ der staatlichen Islamkunde, können an dieser Stelle ausgespart werden, da Islamkunde seinerzeit ausdrücklich als „Platzhalter“ für den Zeitraum eingerichtet wurde, in dem es noch keinen IRU gibt. ${ }^{17}$ Mithin stellten sich für diesen Unterricht auch nicht Fragen, wer Religionsgemeinschaft ist, sowie nach der Besonderheit von Religionsunterricht, Bekenntnisunterricht zu sein und damit Lehren und Erfahrungen zum Inhalt zu haben, die aus Sicht der jeweiligen Religion für wahr und richtig gehalten werden. ${ }^{18}$

Aber auch Islamkunde, die sukzessive von Jahrgangs- zu Jahrgangsstufe dort IRU weichen wird, wo dieser angeboten wird, wurde und wird nicht voraussetzungslos erteilt. ${ }^{19}$ Es entspricht - wie jeder andere Unterricht auch - inhalt-

\footnotetext{
http://www.uni-muenster.de/imperia/md/content/wwu/ab_uni/ab2012/ausgabe03/beitrag_02.pdf (25.11.2012); siehe hierzu auch den Beitrag von Michael Kiefer (S. 211-226) in diesem Sammelband.

${ }^{16}$ Vgl. Klaus Spenlen 2010, S. $222 \mathrm{ff}$.

${ }^{17}$ Vgl. Klaus Spenlen in Klaus Spenlen und Susanne Kröhnert-Othman (Hrsg.), 2012, S. 23-59.

${ }^{18}$ Vgl. Myrian Dietrich 2008, S. 33 ff.

${ }^{19}$ Zwar hat der Islamische Religionsunterricht in NRW zum Schuljahresbeginn 2012/13 erst in 44 von ca. 3.500 Grundschulen und für lediglich 2.500 von insgesamt ca. 320.000 muslimische Schülerinnen und Schüler, ohne Lehrplan und mit nur wenigen ausgebildeten Lehrkräften begonnen.
} 
lich den Verfassungsansprüchen und -prinzipien und dem darauf basierenden Bildungsauftrag von Schule. Für einen an den Maßstäben der Verfassung gemessenen „unaufgeklärten“ Unterricht gibt es in keinem Fall in der Schule eine Legitimation und einen Platz. Den Grundrechten einschließlich des individuellen Rechts, sich nach Art. 4 GG vom Religionsunterricht aus Gewissensgründen abzumelden (Bestandteil der sog. negativen Religionsfreiheit), ist mit Bezug auf den Unterricht ebenso Geltung zu verschaffen wie z. B. dem Demokratie- und Rechtsstaatsgebot. Allerdings gibt es einen zentralen Unterschied zwischen IRU und Islamkunde: Die Länder haben sicher zu stellen, dass im Islamkundeunterricht kein positiver Bekenntnisbezug vorgenommen und damit dem Unterricht kein Bekenntnis verkündender Charakter beigemessen wird.

Gleichwohl können für IRU viele Erfahrungen aus der Islamkunde genutzt werden, etwa bei der Formulierung von Erziehungszielen; der Förderung von Zugehörigkeits- und Minderung von Fremdheitsgefühlen; der Verankerung von Integration als Erziehungsziel der Schule; in unterschiedlichen Werten und Motiven als Stimulus für Lernprozesse. Nutzen sind aber auch in vielen Lehrplaninhalten, in Unterrichtsmaterialien, in den Grundlagen von Akzeptanz bei Eltern, Schülerinnen und Schülern, bei Lehrkräften sowie der ganzen Schulgemeinde für das neue Fach, in gemeinsamem Arbeiten und Abstimmen der Lehrkräfte in schulischen Fachkonferenzen u. a. m. zu erzielen

Zudem kann sich IRU als neues Fach an verbindlichen Inhalten, Methoden, vor allem aber an allgemein akzeptierten Bildungsstandards - möglichst für Doppeljahrgangsstufen - der Islamkunde und anderen Religionsunterrichten orientieren und sie weiterentwickeln. ${ }^{20}$ Denn hier hat IRU einen hohen Nachholbedarf. Während die in den Schulen etablierten Religionen und Religionsge-

Trotzdem sind viele Muslime mit der Abschaffung von Islamkunde zugunsten von islamischem Religionsunterricht nicht einverstanden, insbesondere nicht die „Kulturmuslime“, die eine fundamentale Ausrichtung des Unterrichts durch die islamischen Dachverbände befürchten.

${ }^{20}$ Zum Schuljahr 2012/13 bietet Nordrhein-Westfalen als bevölkerungsreichstes Land der Bundesrepublik Deutschland katholischen, evangelischen sowie lokal begrenzt jüdischen, orthodoxen (serbisch-, syrisch-, griechisch-, rumänisch-, bulgarisch-, russisch-, ukrainisch- und georgisch-orthodoxen), alevitischen und eben neuerdings islamischen Religionsunterricht als ordentliche Unterrichtsfächer an. Die Lehrkräfte im jüdischen und orthodoxen Religionsunterricht sind akademisch ausgebildete Würdenträger ihrer Religion/Konfession und arbeiten im Rahmen von Gestellungsverträgen. 
meinschaften inzwischen länderübergreifende Bildungsstandards für ihren jeweiligen Religionsunterricht entwickelt haben, gilt dies für den islamischen Bereich noch nicht. Gerade die Vielheit des Islam bietet die Chance, die Entwicklung von Bildungsstandards für IRU nicht nur in einer „islaminternen“ Diskussion zu führen, sondern die Tendenzen weiterzuentwickeln, die auf ein interreligiöses und interkulturelles Lernen zielen. ${ }^{21}$

\section{Unterrichtsinhalte}

IRU als bekenntnisorientierter Unterricht wird unspezifisch als religionspädagogisches schulisches Angebot für Muslime verstanden. Da der Islam aber kein monolithischer Block ist und es allein in Deutschland ca. 70-80 Organisationen und Strömungen innerhalb des Islam gibt, ${ }^{22}$ stellt sich die Frage, nach wessen Glaubensgrundsätzen IRU unterrichtet werden soll. Unstreitig ist, dass der Islam in seinen unterschiedlichen Ausprägungen eine Religion bzw. ein Bekenntnis ist. Die Deutsche Islam Konferenz (DIK) hatte bereits 2008 dazu Grundlagen erarbeitet und u. a. festgestellt, dass eine Religionsgemeinschaft auch Angehörige unterschiedlicher, aber verwandter Glaubensbekenntnisse umfassen kann, wie das in denjenigen evangelischen Landeskirchen in Deutschland der Fall ist, die Gemeinden unterschiedlichen Bekenntnisstandes haben (reformiert, lutherisch, uniert). ${ }^{23}$ Welche Bekenntnisse insofern „verwandt“ sind, kann nur unter $\mathrm{Zu}$ grundelegung des Selbstverständnisses der jeweiligen Gemeinschaft bestimmt werden. Religion bzw. Bekenntnis werden durch das religiöse Selbstverständnis der Religionsgemeinschaften konstituiert bzw. definiert. Im Grundsatz können daher muslimische Gemeinschaften selbst darüber entscheiden, ob Angehörige einer bestimmten islamischen Glaubensrichtung ihnen angehören können oder nicht. Die Beschränkung auf die Anerkennung von Koran und Sunna als gemeinsame Glaubensgrundlage reicht aus. Eine weitergehende vollständige kon-

\footnotetext{
${ }^{21}$ Vgl. Gisbert Gemein in Klaus Spenlen und Susanne Kröhnert-Othman (Hrsg.), 2012, S. 61-72.

${ }^{22}$ Vgl. Volker Krech 2009, S. 11.

${ }^{23}$ Vgl. http://www.deutsche-islam-konferenz.de/SharedDocs/Anlagen/DE/DIK/Downloads/Sonstiges/2008-IRU-zwis chenresumee-der-dik, templateld $=$ raw, property $=$ publicationFile.pdf/2008-IRU-zwischenresumee-der-dik. pdf (10.10.2012).
} 
fessionelle Homogenität der Gemeinschaft ist für den Religionsunterricht nicht erforderlich. ${ }^{24}$

Da der konfessionelle Religionsunterricht gem. Art. 7 Abs. 3 Satz 2 GG nach den Grundsätzen der jeweiligen Religionsgemeinschaft gestaltet ist, deren Definition dem Selbstbestimmungsrecht unterliegt, sind mithin auch mehrere islamische Religionsunterrichte unterschiedlicher Bekenntnisse möglich und ggf. rechtlich geboten. Umgekehrt können sich auch Religionsgemeinschaften unterschiedlicher Glaubensrichtungen innerhalb einer Religion zur Formulierung gemeinsamer Grundsätze für einen einheitlichen Religionsunterricht zusammenfinden. In Nordrhein-Westfalen haben die Partner den Weg beschritten, IRU als gemeinsamen Unterricht in deutscher Sprache für alle Ethnien und Glaubensrichtungen des Islam und von in Deutschland ausgebildeten Lehrkräften durchführen und von der Schulaufsicht beaufsichtigen zu lassen.

Ungeachtet unterschiedlicher islamischer Strömungen beschreiben unstreitige didaktische Leitlinien die Themen von IRU: das Verhältnis des Menschen zur Natur, zu anderen Menschen, zu sich selbst und zu Gott. Es geht im IRU also darum, muslimische Kinder und Jugendliche mit islamischen Überzeugungen und Überlieferungen vertraut zu machen; sie mit den religiösen Quellen und deren Botschaften bekannt zu machen; sie zu befähigen, Deutungsmöglichkeiten zu erkennen; sie bei der Entwicklung einer islamischen Identität in einer nichtmuslimischen Umwelt zu unterstützen; ihnen die islamische Traditionen in ihrer Geschichte bewusst zu machen; ihnen Orientierung auf der Suche nach einer eigenen Lebensausrichtung zu geben; ihnen unter Wahrung der eigenen Identität Toleranz gegenüber Andersgläubigen zu vermitteln; sie mit gesellschaftlichen Sachverhalten, wie z. B. religiösem Extremismus, zu konfrontieren; ihre Sprache besonders im Hinblick auf die islamische Sprachkultur und Metaphorik zu fördern; auf der Grundlage islamischer Quellen zu motivieren, eigenverantwortlich zu leben und zu handeln. ${ }^{25}$

Inhalte von IRU sind die Grundlagen der Religion (ușŭl ad dĩn): das Glaubensbekenntnis, die Endlichkeit der Welt, die Geschaffenheit der Welt durch Gott, das Ende der Welt mit der Auferstehung, der Konflikt zwischen Gottes Allmacht

\footnotetext{
${ }^{24}$ Vgl. die Entscheidung des Bundesverwaltungsgerichts vom 23.02.2005 - BVerwG 6 C 2.04.

${ }^{25}$ Vgl. Harry Behr 2005; Irka-Christin Mohr und Michael Kiefer (Hrsg.) 2009.
} 
und Gerechtigkeit. Neben dem Bekanntmachen mit Koran, Hadithe und dem Leben des Propheten lernen die Kinder die fünf Säulen des Islam (ibâdât), also das Glaubensbekenntnis (šahâda. schahâda), das Gebet (şalât), das Almosen (zakât), das Fasten (sawn, saun) sowie die Pilgerfahrt (hadj, haddsch) kennen. Zudem werden sie mit den sechs Glaubensgrundsätze vertraut gemacht: Glaube an Allah, die Propheten, die heiligen Bücher, die Engel, die Vorbestimmung sowie ein Leben nach dem Tod.

Konkrete Ziele, Inhalte, Themen und Methoden beinhalten für die jeweilige Jahrgangsstufe die Richtlinien und Lehrpläne, ${ }^{26}$ die nach den Landesverfassungen bzw. Schulgesetzen in Verantwortung der Länder und in Übereinstimmung mit den Grundsätzen der Religionsgemeinschaften entwickelt werden. ${ }^{27}$ Die Gemeinsamkeit von Staat und Religionsgemeinschaft wird i. d. R. dadurch gesichert, dass die ca. drei bis vier Mitglieder/Lehrkräfte der Lehrplankommission im Einvernehmen mit den Religionsgemeinschaften von der Landesregierung berufen werden. Dabei können von den Religionsgemeinschaften benannte Personen mit theologischer Expertise zusätzlich als Beobachter und Berater an den Sitzungen der Lehrplankommission teilnehmen und bereits dort die Übereinstimmung mit den Grundsätzen der Religionsgemeinschaften herstellen. Abschließend wird die abgestimmte Lehrplan-Fassung von der Landesregierung in ein Beteiligungsverfahren mit allen relevanten gesellschaftlichen Organisationen (u. a. Kirchen, Verbände, Gewerkschaften, Eltern- und Schülervertretungen, Migrantenorganisationen) gegeben. Hier haben die Religionsgemeinschaften und die Dachverbände noch einmal Gelegenheit zur Stellungnahme. Der aufgrund der Voten abschließend überarbeitete Lehrplan wird von der Landesregierung in Kraft gesetzt.

\footnotetext{
${ }^{26}$ Vgl. dazu Hessisches Kultusministerium (Hrsg.) 2012, da es einen Lehrplan in NRW derzeit (Stand: August 2012) noch nicht gibt.

${ }^{27}$ Ausnahmen bilden Baden-Württemberg (§98 SchG) und das Saarland (Art. 29 Saarl. Verf.), wo die Religions-Lehrpläne zwar von den Religionsgemeinschaften entwickelt werden, gleichwohl der Prüfung und Zustimmung des Landes unterliegen.
} 


\section{Lehrkräfte}

Religionsunterricht, für dessen Durchführung eine bestimmte Anzahl von Schülerinnen und Schülern vorhanden sein muss, ${ }^{28}$ kann nur von Personen der Religionsgemeinschaft erteilt werden, die für diese Aufgabe ausgebildet worden sind, islamischer Religionsunterricht mithin ausschließlich von Muslimen. Diese Personen müssen nicht zwangsläufig Landesbedienstete sein. Es gibt verschiedene Optionen, Lehrkräfte für Religionsunterricht zu gewinnen. Ein Blick auf den Religionsunterricht anderer Religionsgemeinschaften gibt hier Aufschluss:

Bei geringen Nachfragequantitäten für Religionsunterricht, etwa weil es an den Schulen wenige Schülerinnen und Schüler für diese Glaubensrichtung gibt (das trifft etwa auf Aleviten zu), erwerben sog. grundständige Lehrkräfte, also Landesbedienstete, die gewillt sind, das Fach zu unterrichten, die notwendigen Qualifikationen in einem Zertifikatskurs. Dass die Personalauswahl im Einvernehmen zwischen dem Land und der Religionsgemeinschaft erfolgt, ist Folge der res mixta.

Eine Alternative hierzu stellen die sog. „Gestellungsverträge“ dar. Hier bildet die Religionsgemeinschaft geeignete Personen, die nicht als Lehrkräfte, sondern etwa als Geistliche arbeiten, für die Aufgaben in den Schulen aus (das trifft etwa auf Juden und Orthodoxe zu). Die Religionsgemeinschaft, die ja den Unterricht in Übereinstimmung mit ihren Grundsätzen - ungeachtet des staatlichen Aufsichtsrechts - verantwortet, benennt dem Land geeignete Personen. Diese Personen werden nicht in den Landesdienst übernommen, das Land refinanziert der Religionsgemeinschaft allerdings die Personalkosten.

Für quantitativ „große“ Religionsgemeinschaften mit der Wahrscheinlichkeit dauerhaften Bestands (etwa Katholiken und Protestanten) bieten die Länder universitäre Ausbildungsgänge im Rahmen der Lehrerausbildung an. Im Anschluss an erfolgreiche Abschlüsse in beiden Staatsexamen - und entsprechendem Bedarf - werden diese Personen als grundständige Lehrkräfte in den Landesdienst eingestellt. Diejenigen Länder, bei denen sich ein relativ großer Bedarf an Lehr-

\footnotetext{
${ }^{28}$ Die jeweilige Schulgesetzgebung des Landes legt fest, wie viele Schülerinnen und Schüler sich für eine Teilnahme am Religionsunterricht einer Glaubensrichtung angemeldet haben müssen, um einen Rechtsanspruch auf Durchführung des Unterrichts zu begründen. In NRW sind dies 12 Schüler pro Schule, vgl. Klaus Spenlen 2008, S. $21 \mathrm{ff}$.
} 
kräften für IRU zeigen wird, werden die Option „Ausbildung zu grundständigen Lehrkräften“ wählen, nicht zuletzt auch, um sich der Verpflichtung von Landesbediensteten zu den Grundwerten des GG zu versichern. Voraussetzung hierfür ist allerdings die Einrichtung entsprechender Lehrstühle bzw. der Ausbau vorhandener.

Zwischenzeitlich, in der IRU noch nicht völlig durch grundständige Lehrkräfte erteilt werden kann, unterrichten diejenigen Islamkundelehrkräfte, die an deutschen Universitäten zu Islamwissenschaftlerinnen und -wissenschaftlern ausgebildet wurden, das neue Fach. Allerdings sollten sie mit einer Zusatzausbildung in schulpädagogischen Fächern sowie in Schwerpunkten bekenntnisorientierten Religionsunterrichts auch dafür qualifiziert werden. Dass in NRW IRU im Schuljahr 2012/13 ohne eine gründliche Nachqualifizierung der Lehrkräfte gestartet ist ${ }^{29}$ diskreditiert das Fach von Beginn an, zumal in den ersten Monaten auch noch nicht einmal ein Lehrplan zur Verfügung steht.

Mit den verschiedenen Optionen sind zwangsläufig unterschiedliche Zeitabläufe verbunden: Die Ausbildung zu grundständigen Lehrkräften bedarf eines langen Atems, sie dauert ca. sechs bis sieben Jahre. Wenn es sich dabei um ein neues Fach für Schulen handelt (wie IRU), beginnt dieser Unterricht in Klasse 1 und steigt Jahr für Jahr einen Jahrgang höher.

Ob Lehrkräfte für IRU notwendigerweise mit Imamen kooperieren müssen, kommt darauf an, ob der Imam zu der von Ceylan klassifizierten kleinen Gruppe der „intellektuell-offensiven“ Imame gehört, die eine kritische Auseinandersetzung mit dem Islam und eine Neuinterpretation des Korans befürworten. ${ }^{30}$ Imame sind in der Regel Vorbeter und Leiter des Gemeinschaftsgebetes, und zudem erfüllen sie als religiöse Repräsentanten der islamischen Gemeinde gottesdienstliche Aufgaben und übernehmen die Gemeindebetreuung, die religiöse und wissenschaftliche Vertretung des Islam nach außen sowie die religiöse Bildung und Erziehung der Gemeindemitglieder. ${ }^{31}$ Darin unterscheiden sie sich in der Wahrnehmung ihrer Aufgaben nicht von Geistlichen der Kirchen. Da die Ausbildung von Imamen an deutschen Universitäten begonnen hat, ist von den

\footnotetext{
${ }^{29}$ Gleichwohl wurde diesen Lehrkräften vom Beirat die schulische Lehrerlaubnis erteilt.

${ }^{30}$ Vgl. Rauf Ceylan 2010, S. 16 ff.

${ }^{31}$ Vgl. Michael Kiefer 2010, S. 185-192.
} 
Studieninhalten, die bekannt sind, zu erwarten, dass eine Zusammenarbeit mit den Schulen für diese, die Gemeinde und das gesamte Wohnumfeld befruchtend sein und zu gelingender Integration sowie zu einem Miteinander über die Religionen hinaus beitragen kann. ${ }^{32}$ Langfristig gesehen sollten die Imamausbildung und die Einrichtung von IRU verbunden werden, da es viele Schnittstellen gibt. Die Forderung nach einer authentischen islamischen Religionspädagogik für Moscheen und Schulen erscheint daher schlüssig. Dennoch sind die beiden Formen der Unterweisung voneinander zu trennen, denn IRU ist „im günstigen Fall komplementär, jedoch grundlegend anders als in Moscheen und Elternhäusern (zu) definieren“ ${ }^{33}$

Das Schlüsselwort für Ansehen und Akzeptanz beider Institutionen, Imamen wie IRU, heißt Transparenz: Dazu sind sog. Tage der offenen Moschee ein guter Anfang, der jedoch zwingend in Dialogen zwischen Schulen, den Imamen, Migrantenorganisationen, interreligiösen Gesprächskreisen, universitären Fakultäten, Studienseminaren u. a. m. über religionswissenschaftliche, fachdidaktische und gesellschaftspolitische Fragen weitergeführt werden muss.

\section{Lehrbücher und Lehrverfahren}

Schulbücher müssen Mindeststandards für Erziehen und Unterrichten in den jeweiligen Jahrgängen vermitteln und sichern. Sie müssen den Aufbau eines progredierenden fachlichen, überfachlichen und methodischen Kompetenzerwerbs unterstützen sowie die Schülerinnen und Schüler in entsprechende Arbeitstechniken einweisen. Dies gilt für jedwedes Unterrichtsfach und jede Jahrgangsstufe und dient dem Ziel, Kindern und Jugendlichen zu ermöglichen, selbständig Lernprozesse $\mathrm{zu}$ organisieren, sukzessive ihre individuellen Lernstände zu vergrößern und die Ziele der jeweiligen Jahrgangsstufen zu erreichen. Wenn die-

\footnotetext{
${ }^{32}$ So bietet die Universität Osnabrück u. a. Religionspädagogik (Moscheepädagogik, Medienpädagogik, Sozialpädagogik, pädagogische Psychologie und Soziologie) und ihre Fachdidaktik, Geschichte der Religionen (Schwerpunkt: Große Weltreligionen), Religionssoziologie (Religion und Modernität, Religion und Globalisierung), Religionspsychologie (religiöse Wahrnehmung und Entwicklung, Religion und Alter, religiöse und psychologische Betreuung der Kranken), Geschichte der muslimischen Migration in Europa und europäische Geschichte, Kultur und Rechtssystem (Organisationsrecht, Staatskirchenrecht) an, vgl. Bülent Ucar, Internetquelle 2010 sowie den Beitrag von Michael Kiefer (S. 211-226) in diesem Sammelband.

${ }^{33}$ Bülent Ucar, Internetquelle 2009, S. 3 ff.
} 
se Mindeststandards zudem auch in Religionsunterrichten verfolgt werden, in denen die Schülerinnen nach Konfessionen getrennten Unterricht erfahren, ist dies die verbindende Klammer, auch mithilfe von Schulbüchern, die in diesen Unterrichten eingesetzt werden, über eine Vergleichbarkeit der Anforderungen gelingende Integration zu fördern.

Allerdings sieht der Islamwissenschaftler Michael Kiefer, dass die islamische Religionspädagogik, die in Deutschland eine sehr junge Fachwissenschaft ist, „[...] an den universitären Standorten [...] seit wenigen Jahren in eher experimentellen Anordnungen gelehrt wird. Ein Hauptproblem ist gewiss darin zu sehen, dass das neue Fach weitgehend von muslimischen Wissenschaftlern vertreten wird, die über keine religionspädagogische Ausbildung verfügen." ${ }^{\text {" Wei- }}$ tere Probleme sieht er in der nicht geklärten Bandbreite des neuen Fachs, also in Fragen, wenn nach sunnitischer Glaubensrichtung unterrichtet werden soll, wie mit den Inhalten anderer Strömungen umgegangen wird und worin sich fachdidaktische Unterschiede zu katholischer oder evangelischer Religionspädagogik äußern. Darin erblickt er aus fachlicher Perspektive für die Produktion von Lehrwerken für IRU ein Wagnis, zumal die Beiräte in NRW und Niedersachsen Einfluss auf die Inhalte nehmen werden. Er resümiert: „Die Schulbuchproduktion für den islamischen Religionsunterricht befindet sich für die nächsten Jahre zwangsläufig in einer Laborsituation, in der es durchaus auch zu Fehlentwicklungen bzw. fragwürdigen Ergebnissen kommen kann.“35

Die folgende Auswahl von Qualitätskriterien für Schulbücher ergibt sich aus den beschriebenen Mindeststandards und entsprechenden Vorgaben in Schulgesetzen. Überschneidungen können nicht ausgeschlossen werden, eine Rangfolge ist nicht beabsichtigt.

Schülerorientierung: Werden Ziel- und Inhaltstransparenz durch die Kapitelüberschriften, die Sequenzialisierung der Kapitel oder durch Offenlegung von Lernzielen erkennbar? Beschränkt sich das Schulbuch in der Anzahl der angebotenen Themen, und geht es stattdessen in die Tiefe und Breite? Werden Alltagserfahrungen und Vorwissen der Schülerinnen und Schüler produktiv

\footnotetext{
${ }^{34}$ Michael Kiefer in Klaus Spenlen und Susanne Kröhnert-Othman (Hrsg.) 2012, S. 99-112.

${ }^{35}$ Ebenda, S. 100.
} 
genutzt? ${ }^{36}$ Werden die Schülerinnen und Schüler aktiviert? Sind Übungsmöglichkeiten vorhanden? Gibt es Differenzierungsangebote, auch solche, die die Schülerinnen und Schüler nicht zugewiesen bekommen, sondern nach Selbsteinschätzung auswählen können? ${ }^{37}$

Fachliche Kompetenzen: Wie wird Islam in den Schulbüchern rekonstruiert? Welcher Islam wird in den Lehrwerken zur Darstellung gebracht (Stichworte: Heterogenität der islamischen Gemeinschaften in Deutschland)? Welche islamischen Quellen finden Verwendung in den Lehrwerken (Stichworte: Koran, Hadîthe, Literatur usw.)? Gibt es erkennbare Kriterien für die Quellenauswahl (Stichworte: sich widersprechende Aussagen in Koran und Sunna, Kontroversität)? Wie werden islamische Quellen präsentiert (Stichworte: Verwendung von dekontextualisierten Zitaten, Quellenangaben)? ${ }^{38}$

Überfachliche Kompetenzen: Unterstützt das Lernmittel die Kinder/Jugendlichen darin, sich unter kultureller Wahrung für ein Leben in der Mehrheitsgesellschaft zu qualifizieren? Wird ein Beitrag geleistet, dass die Kinder/Jugendlichen eine individuelle und gesellschaftliche Persönlichkeit entwickeln? ${ }^{39}$ Lernen die Schülerinnen und Schüler universelle ethische Prinzipien, Normen und Regeln kennen? Werden Angebote gemacht, sich konstruktiv mit diesen Werten Normen und Regeln auseinander zu setzen? Finden Angebote statt, eigene Überzeugungen und Haltungen zu entwickeln? Finden sich hinreichend Angebote zum Aufbau von Sach-, Urteils-, Entscheidungs- und Handlungskompetenz? ${ }^{40}$ Ist das Lernmittel gegenüber unkonventionellen Erscheinungen und unkonventionellem Denken sowie auch gegenüber Minderheiten und Randgruppen tolerant?

\footnotetext{
${ }^{36}$ Vgl. entsprechende Forderungen in folgenden Schulgesetzen - SchG - :§ 4 (1) bremisches Schulgesetz.

${ }^{37}$ SchG: § 3 (1), (2) Hamburg, § 2 (4) Nordrhein-Westfalen, § 4 (1) Schleswig-Holstein, § 1 (1) Rheinland-Pfalz, § 1 (1), (2) Sachsen, §1 (1) Sachsen-Anhalt, § 2 (1) Thüringen, § 4 (2) Mecklenburg-Vorpommern.

${ }^{38}$ Die fachlichen Kompetenzen stammen von Michael Kiefer in Klaus Spenlen und Susanne Kröhnert-Othman (Hrsg.), 2012, S. $100 \mathrm{ff}$.

${ }^{39}$ SchG: § 1 (2) Baden-Württemberg, § 2 (4), (5) Nordrhein-Westfalen, § 1 (1) Saarland, § 4 (3) Schleswig-Holstein, § 1 Berlin, § 4 (4) Bremen, § 5 (3) Bremen, § 1 (2) Rheinland-Pfalz, § 1 (2) Sachsen, § 1 (2) Sachsen-Anhalt, § 2 (1) Thüringen, § 4 (5) Brandenburg, § 2 (1), (2), (3) Mecklenburg-Vorpommern. ${ }^{40}$ SchG: § 1 (2) Baden-Württemberg, § 2 (1) Hamburg, § 1 Berlin, § 5 (3) Bremen, § 1 (2) RheinlandPfalz, § 1 (2) Sachsen-Anhalt, § 2 (2) Mecklenburg-Vorpommern.
} 
Wird mit Werten anderer Kulturkreise bekannt gemacht? Gibt es substantielle Informationen über andere Religionen? Werden Grundlagen geschaffen, andere religiöse Überzeugungen zu respektieren? Berücksichtigt es künftige Anforderungen an die Erkenntnis- und Handlungskompetenzen der Kinder und Jugendlichen? (Wie) Wird sichergestellt, dass alle Schülerinnen und Schüler am Ende ihrer Schulzeit über fachliches, überfachliches und methodisches Basiswissen verfügen?

Methodenkompetenzen: Ist die Anzahl von Materialien zur Themenerarbeitung ausreichend/begrenzt? Werden neben Autorentexten und Dokumenten auch andere Informationsträger wie Statistiken, Tabellen, Karikaturen, Abbildungen etc. eingesetzt? Finden sich hinreichend Angebote zum Aufbau von Methodenkompetenz? Ist das Bildmaterial aktuell, und hat es didaktische Funktion? Finden sich Anregungen zur Einübung genereller und/oder fachspezifischer Arbeitstechniken wie Beobachten, Unterstreichen, Exzerpieren, Ordnen, Nachschlagen, Notieren, Befragen, Vergleichen? Wird in Erarbeitungsformen wie Interview, Befragung, Rollenspiel, Internetrecherche etc. fachlich fundiert eingeübt? Ergänzt das Lehrwerk fachliche um überfachliche, lehrmethodenzentrierte um offene, lehrbuch- und lehrerzentrierte um schülerzentrierte Verfahren?

Lernverfahren: Werden Hilfsmittel für die Erarbeitung eigener Problemlösungen bereitgestellt, oder können lediglich Standpunkte von „Autoritäten“ rezipiert werden ${ }^{41}$ Werden stoffbezogene Verfahren um projektbezogene ergänzt? Wird ausreichend Spielraum für eigene Lösungsansätze der Schülerinnen und Schüler berücksichtigt? Unterstützt das Lernmittel die Erprobung von Gelerntem in variierenden Kontexten? Stellt das Lernmittel breites, lernanschlussfähiges und intelligent nutzbares Orientierungswissen zur Verfügung? ${ }^{42}$ Unterstützt es kumulatives Lernen, indem es z. B. Querverweise herstellt und auf bisher Erlerntes zurückgreift? ${ }^{43}$ Werden Wissensstände miteinander verknüpft und konstruktiv aufgebaut? Führt es hin zur Beherrschung reflexiv einsetzbarer Lern-

\footnotetext{
${ }^{41}$ SchG: § 2 (2) Hessen, § 2 (1) Niedersachsen, § 2 (5) Nordrhein-Westfalen, § 3 (2) Berlin, § 5 (3) Bremen, § 1 (2) Sachsen-Anhalt, § 4 (5) Brandenburg, § 2 (3) Mecklenburg-Vorpommern.

${ }^{42}$ SchG: § 1 (2) Baden-Württemberg, Art 1 (1) Bayern, § 2 (1) Hamburg, § 2 (1) Niedersachsen, § 2 (4) Nordrhein-Westfalen, § 1, 3 (1) Berlin, § 5 (3) Bremen, § 1 (2) Rheinland-Pfalz, § 1 (2) Sachsen, § 2

(1) Thüringen, § 4 (5) Brandenburg, § 2 (2) Mecklenburg-Vorpommern.

${ }^{43}$ SchG: § 2 (6) Nordrhein-Westfalen, § 4 (4) Berlin, § 1 (2) Sachsen, § 4 (6) Brandenburg.
} 
strategien/Methoden? Werden Lernprozesse progredierend, d. h. mit zunehmendem Lernzuwachs aufgebaut? Sind die Fragen auf Verständnis hin angelegt oder ausschließlich auf Merkwissen gerichtet, sind sie anregend und aufschlussreich für die Schülerinnen und Schüler oder bleiben sie abstrakt?

Gesellschaftsorientierung: Wird das, was in der Gesellschaft strittig ist, auch im Schulbuch als strittig dargestellt? Befähigt das Lehrwerk, sich mit autoritaristischen Argumentationen kritisch auseinanderzusetzen und darauf zu reagieren? Werden Wertungen vorweggenommen, oder ist das Lernmittel offen angelegt? Reproduziert das Unterrichtswerk Rollenklischees, insbesondere die stereotype Zuweisung von „typisch männlichen“ und „typisch weiblichen“ Eigenschaften und Verhaltensweisen? ${ }^{44}$ Nimmt das Werk Identifikationsangebote für Schülerinnen auf, die Mädchen und Frauen nicht einseitig auf traditionelle sowie benachteiligende Rollen in Familie, Beruf und Gesellschaft verpflichten, sondern diese als selbstständige, verantwortliche und kreativ handelnde Personen zeigen $?^{45}$ Nimmt das Unterrichtswerk Identifikationsangebote für Schüler auf, die zur Auseinandersetzung mit der Männerrolle in unserer Gesellschaft anregen?

Die bekanntesten Schulbücher für Islamkunde ${ }^{46}$ die zukünftig auch im neuen Schulfach IRU eingesetzt werden, wurden im Hinblick auf die genannten Standards hin bereits analysiert und besprochen, die Stärken und Schwächen Kriterien orientiert herausgearbeitet. ${ }^{47}$ Auf diese Untersuchungen wird an dieser Stelle verwiesen, weil eine detaillierte Kritik an den Schulbüchern den Rahmen dieses Beitrags sprengen würde.

Eine Folge der Entscheidung für die Einführung von IRU bezieht sich - über erste Versuche in Grundschulen hinaus - auf die Qualität der Textanalysen im Unterricht. In der Schule muss unverzichtbar die historisch-kritische Be-

\footnotetext{
${ }^{44}$ SchG: Art 2 (1) Bayern, § 2 (1) Hamburg, § 2 (2) Hessen, § 2 (1) Niedersachsen, § 2 (6) NordrheinWestfalen, § 4 (2) Schleswig-Holstein, § 1 Berlin, § 5 (2) Bremen, § 1 (2) Rheinland-Pfalz, § 1 (2) Sachsen-Anhalt, § 2 (1) Thüringen, § 4 (5) Brandenburg, § 2 (3) Mecklenburg-Vorpommern.

${ }^{45}$ Wird das Berufsspektrum von Mädchen und Frauen erweitert? Werden die Leistungen der Frauen in Wissenschaft und Gesellschaft gewürdigt? Wird den Benachteiligungen von Mädchen und Frauen aktiv entgegengewirkt? Vgl. dazu folgende Schulgesetze: § 2 (2) Hessen, § 3 (3) Berlin, § 4 (4) und (5) Brandenburg; Art 2 (1) des bayerischen Gesetzes über das Erziehungs- und Unterrichtswesen.

${ }^{46}$ Vgl. die Schulbücher in der abschließenden Literaturliste.

${ }^{47}$ Vgl. Klaus Spenlen und Susanne Kröhnert-Othman (Hrsg.) 2012.
} 
trachtung von Texten jedweder Art, mithin auch der von Koran und Sunna, Grundlage sein, bei der exklusive Wahrheitsansprüche inakzeptabel sind. ${ }^{48}$ Dieses Selbstverständnis geht nicht mit einem Verzicht auf islamische Tradition einher, denn in der Tradition des Islam gibt es hinreichend Belege, Texte für alle Zeiten nicht einfach wörtlich zu übernehmen, sondern in ihren jeweiligen Kontexten zu bestimmen.

Daraus folgt notwendigerweise, dass es eine Reihe von Interpretationsmöglichkeiten gibt, je nach dem Stand menschlichen Wissens und nach den jeweiligen sozio-politischen und kulturellen Umständen der jeweiligen Interpreten. Ausdrücke und Verse müssen mehrere Bedeutungsebenen besitzen, denn es handelt sich um Aussagen, die sowohl eine konkrete Bedeutung für ihre ursprünglichen Hörer gehabt haben als auch eine Bedeutung, die spätere Zuhörer aus ihnen herauslesen können. So wird sich an vielen Stellen des Korans die Frage stellen, ob sie neben ihrer wörtlichen Bedeutung eine metaphorische Bedeutung besitzen, und wenn ja, welche. ${ }^{49}$

Gerade das Ernstnehmen der Interessen und Voraussetzungen von Schülerinnen und Schülern eröffnet Chancen, unterschiedliche „Bindungskräfte des Islam für Jugendliche, die Diskrepanzen zwischen Anspruch religiöser Regeln und der Praxis des Alltagslebens etc." zu thematisieren und heraus zu arbeiten, was Islam für Jugendliche bedeutet. ${ }^{50}$ Auch die Scharia (arab.: šarî’a), das islamische kanonische Recht, sei eine Konstruktion der Nachwelt Mohammeds und in ihrem Gesamtumfang heute nicht mehr akzeptabel, so der renommierte bosnische Kultur- und Religionswissenschaftler Balic. ${ }^{51}$ „Der dynamische Charakter (von Koran und Sunna, Anmerkung des Verfassers) bleibt nur erhalten, wenn eine fortwährende Interpretation für eine textlich zwar abgeschlossene, jedoch in ihrer Anwendung fortlaufende Offenbarung möglich ist. Allein die Bemühungen

\footnotetext{
${ }^{48}$ Das Verständnis von „historisch-kritischer Betrachtung von Texten“ beinhaltet im Sinne Oemings das „hermeneutischen Viereck“, dem außer der historisch-kritischen die sozialgeschichtliche Exegese und zudem die historische Psychologie sowie die „New Archaeology“ angehören, also Methoden, die Texte über Personen, Sachverhalte und Ereignisse in ihrer Zeit sehen und diese in ihren historisch-soziologischen Kontexten reflektieren. Dabei nehmen die Vor- und Entstehungsgeschichte der Texte, ihre regionale Verortung etc. ebenso wichtige Positionen ein wie archäologische Erkenntnisse und psychologische Profile. Vgl. hierzu Manfred Oeming 2010.

${ }^{49}$ Nasr Hamid Abu Zaid 2011, S. 78.

${ }^{50}$ Wolfram Weisse 2008, S. 2.

${ }^{51}$ Vgl. Smail Balic 2001.
} 
darum stellen in der Praxis weit mehr als einen iğtihad dar" ${ }^{52}$ Es ist nicht nur möglich, sondern unverzichtbar, „ein und denselben Vers geschlechtergerecht oder Frauen marginalisierend zu übertragen " ${ }^{33}$ - oder aber unter anderen Kriterien zu interpretieren.

\section{Ausblick}

Als zentrale Fragen verbleibt die Reflexion von Sachverhalten, die IRU in den Blick des „gesellschaftlichen Wunschbildes Deutschland“ rückt.

Integrationsvorstellungen realisieren sich nicht nur in Familien und in Schulen, sondern auch in mehr oder weniger aktiven Integrationsbemühungen der Aufnahmegesellschaft. Zu den Störfaktoren gelingender Integration zählen u. a. die Arbeitsmarktkrise. Arbeitslosigkeit und die Auslagerung minderqualifizierter Arbeitskraft, sie haben eine der zentralen Integrationsinstanzen, das Berufsfeld, einbrechen lassen. Hinzu kommen soziale Polarisierungstendenzen, nicht zuletzt verschärft durch die Erosion der Sozialsysteme sowie die Betroffenheit breiter Bevölkerungsteile von „Down-Sizing“.

Die Unterschiede in der Religiosität von Muslimen in Deutschland und der der nichtmuslimischen Bevölkerung mag zu dem Eindruck beitragen, dass seit den 1990er Jahren in ganz unterschiedlichen Alltagsbereichen gelingende Integration bei Muslimen zu regredieren scheint. Indikatoren dafür sind eine $\mathrm{Zu}-$ nahme öffentlich gelebter Religion, gemessen an Moscheebesuchen, Bedeckung von Frauen, „islamischen Sonderwegen“ in den Schulen u. a. m. ${ }^{54}$ Zudem ist ein Rückgang im Gebrauch der deutschen Sprache, eine Zunahme im Konsum „islamischer" Medien ${ }^{55}$ sowie Veränderungen in der jugendlichen Musikkultur ${ }^{56}$

\footnotetext{
${ }^{52}$ Rabea Müller 2008, S. 16. Iğtihad ist das arabische Wort für Anstrengung und meint die weitgehend selbständige Auslegung des Koran. Müller verweist an dieser Stelle zudem auf „klassische Herangehensweisen an den Text", die im Allgemeinen „über die wesentliche linguistische Bedeutung (al-haqiqa al-lugawiyya), die traditionelle Bedeutung (al-haqiqa al-urfi yya) und die Bedeutung gemäß der Scharia (al-haqiqa al-šariyya) (erfolgt). Es ist möglich, den Texten durch die Standardisierung nach der Zeichensetzung (nuțq), der Setzung der diakritischen Zeichen (šakl) und der Lesung (qira'a) nachzugehen."

${ }^{53}$ Ebenda.

${ }^{54}$ Vgl. Klaus Spenlen 2009, S. $332 \mathrm{ff}$.

${ }^{55}$ Vgl. Helmut Reitze 2010 sowie BMI 2012, S. $379 \mathrm{ff}$.

${ }^{56}$ Vgl. den Beitrag von Martin Seeliger/Marc Dietrich (S. 251-272) in diesem Sammelband.
} 
als Ausdruck veränderter Orientierungen feststellbar. Diese Faktoren scheinen Nährboden für eine Verfestigung tendenziell integrationsresistenter Sozialisationsmilieus zu sein, in denen ein Teil der muslimischen Kinder und Jugendlichen zunehmend religiös-autoritär, patriarchalisch und antiemanzipatorisch erzogen wird. Hier kann IRU eine gesellschaftlich bedeutsame Befriedungsleistung erbringen.

Integration muss wechselseitig gedacht und immer wieder neu als gesellschaftliches Projekt verstanden werden, das alle Menschen in Deutschland angeht, Eingewanderte wie Einheimische, Nichtgläubige wie Gläubige, Christen wie Muslime. Es ist zunehmend schwierig, sich eine junge Generation vorzustellen, in der sich „die“ Muslime und „die“ Nichtmuslime gegenseitig als Kollektive gegenüberstehen, von denen gegenseitige Anpassungsleistungen gefordert werden. Notwendig erscheint vielmehr gesellschaftliche Kohäsion, insbesondere nach bestehenden und zu vermittelnden Gemeinsamkeiten verbindlicher Wertvorstellungen, die die Gesellschaft zusammenhält. Alle müssen ihren Integrationsbeitrag leisten, ihn nicht nur der Politik überlassen, die für sich Religionsgovernance reklamiert und am liebsten auch Religionsfragen regeln möchte. Alle gesellschaftlichen Kräfte müssen sich gemeinsam betätigen, gemeinsam handeln, zusammen auf die gemeinsame Umwelt einwirken können. ${ }^{57}$ Dabei müssen Positionen überdacht werden, denn wer sich als der oder die „Andere“ denkt, kann sich kaum auf eine Perspektive von Gemeinsamkeiten oder Ähnlichkeiten einlassen. Erwartet werden darf von Muslimen wie Nichtmuslimen, die Gratwanderung der Bestimmung des „Eigenen“ und des „Anderen“ im Sinne von Gemeinsamkeiten zu reflektieren und sie nicht bei Bedarf wechselseitig strategisch einzusetzen, um pauschale Zumutungen und Vorwürfe gegenüber der Gruppe zu formulieren und abzuwehren oder Loyalitäten von Gruppenmitgliedern einzufordern. Erwartet werden darf aber auch ein modernes, plurales Islamverständnis und damit zugleich eine Abkehr vom Mainstream-Islam der Verbände. Und erwartet werden darf außerdem, dass Vertreter des Islam regen Kontakt in der Kommune pflegen, weil der unverzichtbar für wechselseitige Informationen, Transparenz und Offenheit ist. Eine erfolgreiche Integrationspo-

\footnotetext{
${ }^{57}$ Vgl. die Ausführungen zum Beitrag der Zivilgesellschaft am Integrationsprozess von Zuwanderern bei Rita Süssmuth 2006 .
} 
litik muss diesen wechselseitigen Prozess und die mit ihm verbundenen Ängste durch differenzierte und abgestimmte Informationen fördernd, aber auch fordernd begleiten.

Dieser Prozess wird auch für IRU weder kurz- noch mittelfristig abgeschlossen werden können. Wenn Integration in der Schule allerdings als offener Prozess verstanden wird, in dessen Verlauf Lehrkräfte, Schülerinnen und Schüler wie deren Eltern nach immer neuen Wegen suchen, auf Diversität zu antworten, ist das bereits eine solide Basis. Wenn zudem das Eigene nicht überbetont und Gemeinsames als vorrangig betrachtet wird, können damit Identitätsbildung und Sozialbindungen entscheidend gefördert werden. Damit wären zugleich die entscheidenden Voraussetzungen eines staatlich verantworteten Unterrichts geschaffen, dessen Ziel die Bildung eines reflektierenden kritischen Individuums und seiner Handlungskompetenzen, nicht aber die Herausbildung identitätspolitischer Positionen eines Kollektivs von Muslimen wäre.

Allerdings wird bislang bei der Frage, ob IRU die Integration von Muslimen fördert oder eher behindert, gewissermaßen stillschweigend davon ausgegangen, dass Ziele, Inhalte und Verfahren von IRU nicht im prinzipiellen Widerspruch zu den Grundlagen der staatlichen Ordnung stehen, im Idealfall im Unterricht selbst Verfassungsgrundsätze vermittelt werden. Unstreitig ist Bestandteil von schulischem Unterricht, der nicht Religionsunterricht ist, im Sinne der Grundwerte des Grundgesetzes zu erziehen und ein gutes Zusammenleben der Menschen in Gleichberechtigung, Frieden und gegenseitiger Achtung zu fördern. Ob das auch für Religionsunterricht, hier IRU, gilt, ,setzt die Klärung der Frage voraus, wie die Rechte für die Ausgestaltung des Religionsunterrichts zwischen Staat und Religionsunterricht verteilt werden" ${ }^{58}$ Konkret heißt das: Wie gehen IRU und Schulaufsicht mit der Frage der Gleichstellung von Mann und Frau um, der positiven wie negativen Religionsfreiheit, mit koranischen Züchtigungen und Strafen sowie weiteren Konfliktlinien der Scharia zur Werteordnung der Bundesrepublik Deutschland?

Die Richtung des Verfassungskonflikts zwischen der Religionsfreiheit und dem Aufsichtsrecht des Staates über das Schulwesen ist noch nicht abgeschlossen. Es muss dahin entschieden werden, dass „Kriterien für die Abwägung von

\footnotetext{
${ }^{58}$ Hans Markus Heimann 2011, S. 58.
} 
Art. 4 Abs. 1 und 2 GG und den sonstigen Verfassungsgütern, über die das staatliche Aufsichtsrecht eine inhaltliche Überprüfung des Religionsunterrichts rechtfertigen kann" ${ }^{59}$ entwickelt werden. Und das spätestens bis zu dem Zeitpunkt, zu dem IRU nicht nur in der Primar-, sondern aufsteigend auch in der Sekundarstufe unterrichtet wird, weil dann die „antagonistischen Energien “60 in Unterrichtsinhalten deutlicher hervortreten.

Gemeinsame Kriterien könnten im Konzept der Mündigkeit gefunden werden, das konkret im sog. Beutelsbacher Konsens zum Ausdruck kommt [das Überwältigungs- bzw. Indoktrinationsverbot; das Kontroversitätsprinzip („Was in Wissenschaft und Politik kontrovers ist, muss auch im Unterricht kontrovers erscheinen"); die Hinführung zur selbstständigen Interessenwahrnehmung (,eigene Interessenlage analysieren und die vorgefundene Lage im Sinne eigener Interessen beeinflussen lernen")]. ${ }^{61}$ Für Religionsunterricht konkretisiert sich das Konzept in den Grundsätzen Kunde vor Verkündigung; Kenntnisse vor Bekenntnissen; Aushandlung theologischer Positionen sowie Diskursivität als Unterrichtsprinzip. Dass diese Grundsätze realisiert werden, ist Aufgabe der Schulaufsicht.

$\mathrm{Ob}$ zu den Aufgaben von IRU auch explizit die von politischer Seite geforderte Extremismusprävention gehört, wird sich möglicherweise dauerhaft ebenfalls mit der im Unterricht zu vermittelten Wertschätzung für die demokratische Grundordnung erledigen.

Die Mitwirkung der Religionsgemeinschaften beim Religionsunterricht erschöpft sich aber nicht in der Festlegung der inhaltlichen Grundsätze des Religionsunterrichts. Vielmehr haben sie auch das Recht zu entscheiden, ob eine Lehrkraft Religionsunterricht ihrer Konfession erteilen darf. Diese Entscheidung wird bei den christlichen Kirchen in Form der erforderlichen kirchlichen vocatio bzw. missio canonica getroffen. Auch beim IRU muss sichergestellt werden, dass aufgrund klarer Vertretungsregelungen die für die Erteilung der iĝaza genannten Lehrerlaubnis zuständigen Organe benannt werden. Das gleiche gilt für die universitäre Lehrerlaubnis nihil obstat. Beide Lehrerlaubnisse wird in NRW für

\footnotetext{
${ }^{59}$ Ebenda, S. 64.

${ }^{60}$ Jan Assmann 2003, S. $28 \mathrm{ff}$.

${ }^{61}$ Siegfried Schiele u. a. 1977, S. $179 \mathrm{ff}$.
} 
die Übergangszeit bis Ende Juli 2019 der Beirat erteilen, der sich dafür bereits Anfang 2012 eine Geschäftsordnung gegeben hat, die sich an entsprechenden Grundlagen der Kirchen orientiert. ${ }^{62}$ Und innermuslimische Konflikte zeichnen sich dabei bereits jetzt ab, da sich das konservative Islamverständnis der Dachverbände im Beirat auch an Vorstellungen von Lebensgestaltung der Lehrkräfte reibt, die in Schulen und Universitäten islamische Religion bzw. Theologie lehren. ${ }^{63}$

Mit der Implementation von IRU muss zudem die Frage gelöst werden, wie die Zivilgesellschaft (Bürgerinnen und Bürger, Bürgerinitiativen, Vereine, Gewerkschaften, Wohlfahrtsverbände, Stiftungen, NGO- und viele weitere Gruppierungen) in den Dialog um die Zukunft bekenntnisorientierten Religionsunterrichts eingebunden werden kann. Deren Bedeutung für die Integrationsarbeit bildete sich schon lange, bevor die Politik Deutschland als Einwanderungsland anerkannte, heraus, und deren Aktivitäten haben meinungsbildend gewirkt, indem sich ihre Vertreterinnen und Vertreter eingemischt und Öffentlichkeit geschaffen haben. Die in Umfragen immer wiederkehrenden Vorbehalte gegen „den Islam“ legen es nahe, diese Erfahrungen der Zivilgesellschaft zu nutzen und Muslime wie Nicht-Muslime zeitnah über Ziele, Themen, Lerninhalte sowie Lehrkräfte von IRU zu informieren. IRU muss als Bestandteil gelingender Integration verstanden und kommuniziert werden. In diesen Prozess müssen weite Bevölkerungsteile aktiv und konstruktiv - lokal, regional, übergreifend - eingebunden, und die Parteien- und Verbändedemokratie muss in gleichem Maße eingedämmt werden. Ohne die Zivilgesellschaft und in besonderer Weise ohne Muslime in Deutschland können die positiven Aspekte, die von IRU ausgehen können, nicht sichtbar werden. Primäres Ziel nicht in den Dachverbänden organisierter Musliminnen und Muslime ist „nicht die Gleichstellung der Verbände

\footnotetext{
${ }^{62}$ Allerdings sind die Grundlagen der missio canonica und der vocatio transparent und jedermann zugänglich, für die iĝaza verweigern hingegen die Verbände und die Ministeriumsvertreter im Beirat deren Veröffentlichung. Bekannt geworden ist jedoch ein ca. 30-minütiges Gespräch, das die Lehrkräfte über sich ergehen lassen müssen, in dem u. a. die Loyalität zum Beitrat (!) abgeklopft wird.

${ }^{63}$ Vgl. exemplarisch die Artikel von Martin Spiewak, Glaubenszeugnis. Unter den Muslimen ist ein Streit darüber ausgebrochen, wer ihre Religion an Schulen unterrichten darf, in: DIE ZEIT Nr. 16 vom 12.04.2012, S. 34 sowie von Hermann Horstkotte, Auf dem Weg zum Staatsislam, in: Frankfurter Allgemeine Zeitung vom 25. Juli 2012, Nr. 171, S. 5.
} 
und Vereine mit den christlichen Kirchen“, sondern eine „grundsätzliche Auseinandersetzung“ mit „den islamistischen Verbänden und Vereinen, und zwar mit dem Ziel, sie und ihre Anhänger davon zu überzeugen, dass die Gesamtheit der demokratischen Werte und Normen von allen, die in dieser Gesellschaft leben, uneingeschränkt zu akzeptieren ist, und religiös begründete Partikularforderungen nur innerhalb dieses Rahmens eine Geltung haben können"64 .

\section{Literatur}

Abu Zaid, Nasr Hamid mit Sezgin, Hilal, Der Koran und die Zukunft des Islam. Die Basis einer Weltreligion, Freiburg 2011.

Anschütz, Gerhard, Weimarer Reichsverfassung, Berlin 1933.

Assmann, Jan, Die Mosaische Unterscheidung oder der Preis des Monotheismus, München 2003.

Balic, Smail, Islam für Europa. Neue Perspektiven einer alten Religion, Köln 2001.

Behr, Harry, Curriculum Islamunterricht. Analyse von Lehrplanentwürfen für islamischen Religionsunterricht in der Grundschule. Ein Beitrag zur Lehrplantheorie des Islamunterrichts im Kontext der praxeologischen Dimension islamisch-theologischen Denkens, Bayreuth 2005.

BAMF - Bundesamt für Migration und Flüchtlinge (Hrsg.), Muslimisches Leben in Deutschland. Im Auftrag der Deutschen Islamkonferenz, Nürnberg 2009.

BMI - Bundesministerium des Innern (Hrsg.), Muslime in Deutschland, Hamburg 2007.

Dass., Bundesministerium des Innern (Hrsg.), Lebenswelten junger Muslime in Deutschland. Ein sozial- und medienwissenschaftliches System zur Analyse, Bewertung und Prävention islamistischer Radikalisierungsprozesse junger Menschen in Deutschland, Bonn 2011, veröffentlicht 2012.

Ceylan, Rauf, Die Prediger des Islam. Imame - wer sie sind und was sie wirklich wollen, Bonn 2010.

Dietrich, Myrian, Islamischer Religionsunterricht. Rechtliche Perspektiven, in: Axel Frhr. Von Campenhausen u. a. (Hrsg.), Schriften zum Staatskirchenrecht, Band 31, Frankfurt a. M. 2006.

Dies., Von der Islamkunde zum Islamischen Religionsunterricht: Wo liegt der rechtliche Unterschied?, in: Kiefer, Michael, Gottwald, Eckart und Ucar, Bülent (Hrsg.), Auf dem Weg zum Islamischen Religionsunterricht. Sachstand und Perspektiven in NordrheinWestfalen, Münster 2008, S. 33-44.

${ }^{64}$ Vgl. exemplarisch die Ziffern 3 und 7 der Plattform der Initiative von säkularen und laizistischen Bürgerinnen und Bürger aus islamisch geprägten Herkunftsländern in Hessen, derzeit ohne URL, sondern lediglich als Printversion. 
Heimann, Hans Markus, Islamischer Religionsunterricht und Integration, Münster 2011. Hessisches Kultusministerium (Hrsg.), Bildungsstandards und Inhaltsfelder. Das neue Kerncurriculum für Hessen. Primarstufe - Entwurf - Islamische Religion, Wiesbaden 2012.

Kiefer, Michael, Zielsetzungen einer Imamausbildung in Deutschland - Vom einfachen Vorbeter zum multifunktionalen Akteur?, in: Ucar, Bülent (Hrsg.), Imamausbildung in Deutschland. Islamische Theologie im europäischen Kontext, Göttingen 2010.

Krech, Volker, Islam und Integration - 12 Thesen, in: Friedrich Ebert Stiftung (Hrsg.), Migration-Integration-Religion, in: Policy Politische Akademie Nr. 30, Berlin 2009.

Mohr, Irka-Christin und Kiefer, Michael (Hrsg.), Islamunterricht - Islamischer Religionsunterricht - Islamkunde. Viele Titel - ein Fach?, Bielefeld 2009.

Müller, Rabea, in: Barlas, Asma u. a., Der Koran neu gelesen: feministische Interpretationen, in: Friedrich-Ebert-Stiftung (Hrsg.), Dokumentation. Politische Akademie/Interkultureller Dialog. Islam und Gesellschaft Nr. 6, Berlin 2008.

Oeming, Manfred, Biblische Hermeneutik, Darmstadt 2010.

Reitze, Helmut (Hrsg.), Media Perspektiven Basisdaten. Daten der Mediensituation in Deutschland 2010, Frankfurt a. M. 2010.

Rhode, Ulrich, Rechtliche Anerkennung der muslimischen Glaubensgemeinschaften als Religionsgemeinschaften: juristische Aspekte, in: Kurt, Hüseyin, Weber, Edmund u. a. (Hrsg.), Die Zukunft der Muslime in Deutschland, Frankfurt a. M. 2011, S. 111-118.

Schiele, Siegfried u. a., Das Konsensproblem in der Politischen Bildung, Stuttgart 1977.

Spenlen, Jeannette, Gender-Perspektiven „muslimischer Erziehung“, in: Orientierungen. Zeitschrift zur Kultur Asiens, Nr. 2, Bonn 2009, S. 30-69.

Spenlen, Klaus, Auf dem Weg zu islamischem Religionsunterricht - Chancen, Grenzen sowie Lösungsversuche der Länder, in: Kiefer, Michael, Gottwald, Eckart und Ucar, Bülent (Hrsg.), Auf dem Weg zum Islamischen Religionsunterricht. Sachstand und Perspektiven in Nordrhein-Westfalen, Münster 2008, S. 21-32.

Ders., Integration muslimischer Schülerinnen und Schüler. Analyse pädagogischer, politischer und rechtlicher Faktoren, Münster 2010.

Ders. und Kröhnert-Othman, Susanne (Hrsg.), Integrationsmedium Schulbuch. Anforderungen an islamischen Religionsunterricht und seine Bildungsmaterialien, in: Eckert. Die Schriftenreihe, Band 132, Göttingen 2012.

Süssmuth, Rita, Migration und Integration: Testfall für unsere Gesellschaft, München 2006.

Weisse, Wolfram, Islamischer Religionsunterricht in Deutschland - ein Beitrag zur Integration? Religionspädagogischer Kommentar mit Bezug zu Alternativen in Deutschland und Europa, Berlin 2008. 


\section{Schulbücher}

Abdel-Rahman, Annett und Ulfat, Fahimah, Bismillah - Wir entdecken den Islam, Arbeitshefte für den Islamunterricht, Klassen 1/2, 3 und 4, Braunschweig 2011.

Erkan, Serap u. a., Mein Islambuch. Grundschule 1/2, München 2009.

Gebauer, Klaus, Yakar, Havva u. a., Die schöne Quelle. Islamunterricht in der Grundschule. Klasse 3: helfen und stärken, Köln (2008).

Dies., Die schöne Quelle. Islamunterricht in der Grundschule. Klasse 4: Teilen und Anteil nehmen, Köln 2011.

Kaddor, Lamya u. a. (Hrsg.), Saphir 5/6. Religionsbuch für junge Musliminnen und Muslime, München 2008.

Dies., Saphir 7/8. Religionsbuch für junge Musliminnen und Muslime, München 2011.

Ucar, Bülent (Hrsg.), EinBlick in den Islam. Ein Schulbuch für die Jahrgangsstufe 5/6, Hückelhoven 2010.

Ders., EinBlick in den Islam. Ein Schulbuch für die Jahrgangsstufe 7/8, Hückelhoven 2011.

\section{Internetquellen}

Czermak, Gerhard, Das System der Religionsverfassung des Grundgesetzes, 2002, unter: forschungsgruppe weltanschauungen in deutschland: http://fowid.de/fileadmin/textar chiv/Czermak_Gerhard/Religionsverfassungsrecht_im_Grundgesetz_TA2000_2.pdf (28.08.2012).

Ucar, Bülent, Die Bedeutung und Entwicklung der Islamischen Religionspädagogik in der Schule und in den Gemeinden, Vortrag am 27.06.2009 in der Akademie Hofgeismar, unter: http://www.ekkw.de/akademie.hofgeismar/publ/Vortraege/Neu\%20ab\%202009/ 09081_Vortrag\%20Prof $\% 20 \% 20$ Buelent $\% 20$ Ucarlslamische $\% 20$ Religionspaedagogik. $\operatorname{pdf}(28.08 .2012)$.

Ders., Die Gelehrten sind die Erben der Propheten: Auf dem Weg zu einer Imamausbildung an der Universität Osnabrück, erstmalig veröffentlicht im Webportal der Heinrich-Böll-Stiftung 2010, aktuell unter: http://www.ufuq.de/newsblog/1051-die -gelehrten-sind-die-erben-der-propheten-auf-dem-weg-zu-einer-imamausbildung-an-der -universitaet-osnabrueck-von-buelent-ucar (28.08.2012). 